\title{
Dynamic torsional buckling: prebuckling waves and delayed instability
}

\author{
X.W. Zhao*t \& G.H.M. van der Heijden ${ }^{\dagger}$
}

October 19, 2018

\begin{abstract}
We study torsional buckling of a rod within the dynamics context, recognising that in a real experiment a twisting moment is not instantaneously applied and therefore an angular velocity (a spin) always accompanies a twist. We derive and solve the wave equation that governs prebuckling torsion dynamics and highlight the compatibility problem between initial and boundary conditions (corner singularity) plaguing numerical solution of the equation. We deal with this problem by introducing a smoothing function. Prebuckling torque oscillations are a major concern in various turbine applications.

Torsional instability, upon further increase of the applied moment, is found to be delayed by the dynamic loading. We determine the dependence of the critical load on the rate of application of the moment by computing initial postbuckling solutions and extrapolating back to the critical point. For these computations we use the geometrically-exact Cosserat rod equations, which we discretise with the generalised- $\alpha$ method. We argue that in addition to inertia a gyroscopic effect may play a role in the delay. Our results may help explain delayed torsional buckling recently observed in simulation studies of flexible marine risers.
\end{abstract}

Keywords: Cosserat rod, torsional wave, torsional buckling, delay of buckling, corner singularity

\section{Introduction}

The instability of an elastic rod at a critical value of an applied end twisting moment is a classical problem in mechanics. It is good to realise though that pure static moments are hard to produce in practice. Such moments are applied by twisting one end of the rod over a finite time, so that the static moment is always accompanied by an angular velocity and a generation of waves. Here we study this torsional buckling problem as a true dynamical problem. We are particularly interested in the effect the rate of application of the twist has on the critical torsional load.

Most of the previous work on torsional buckling is focused on static deformation. Thompson and Champneys [24] studied helical and localising modes in tensile torsional buckling with experimental validation. Torsional buckling under a compressive load was studied by Atanackovic and

\footnotetext{
${ }^{*}$ School of Aerospace Engineering and Applied Mechanics, Tongji University, Shanghai, China

${ }^{\dagger}$ Department of Civil, Environmental \& Geomatic Engineering, University College London, London WC1E 6BT, UK (e-mail: xingwei.zhao@ucl.ac.uk, g.heijden@ucl.ac.uk)
} 
Glavardanov [2] and Glavardanov and Maretic [7] who also considered the effects of shear and axial compressibility. Goss et al. [9] and van der Heijden et al. [26] studied torsional instability and post-buckling behaviour, including self-contact, both theoretically and experimentally.

Rod dynamics has also been well studied. One of the first computational formulations for geometrically-exact rod dynamics was that of Simo and Vu-Quoc [22]. Torsional vibrations were studied in [25] in a Cosserat model for drillstring dynamics. The Cosserat rod dynamics equations were also used in [23] for hair modelling. Dynamical instabilities were studied in [8, 16]. Loop formation in twisted rods was studied in [11]. In [10] torsional buckling was considered, but twist rates were deliberately taken very small in order to approximate closely the static critical load. The effect of the twist rate on the critical load was not investigated. Indeed, compared to the statics case reviewed above, dynamical buckling has not received much attention. In [15] column buckling due to axial impact is studied, but this is a different kind of buckling problem. There have been a few recent studies on column buckling under a gradually increasing compressive load (e.g., [17], where an increase of the critical load from the classical static Euler load is found), but there appear to be no similar studies on torsional buckling.

The analytical approach of [17] is not available for our torsional case because the applied load appears in the boundary conditions and not in the equation. We therefore resort to a numerical approach in which we compute the initial postbuckling response and then extrapolate back to the critical buckling point. The postbuckling response requires the solution of nonlinear largedeformation rod equations. We use the geometrically-exact Cosserat formulation with linearly elastic material behaviour. The rod is assumed to be inextensible and unshearable. The purpose of this paper is to study the effect of dynamic loading, both on the prebuckling travelling-wave solution and on the torsional buckling instability.

The outline of this paper is a follows. The Cosserat rod dynamics formulation is presented in Section 2, where the equations are developed in the body frame. For the numerical solution we use a discretisation based on the generalised- $\alpha$ method [4], which is outlined in Section 3. Section 4 discusses the postprocessing of solutions required for computing postbuckling rod shapes. In Section 5 we first derive the linear wave equation governing prebuckling waves as a result of the dynamic loading. We construct the exact solution of an appropriate initial-boundary-value problem for this wave equation and use this to highlight the appparently little-known incompatibility between common (and physical) initial and boundary conditions in the dynamics context. This incompatibility prevents a straightforward comparison between analytical and numerical solutions, which is only possible after applying a smoothing function to the dynamic boundary condition. We then, in Section 6, investigate the effect that the rate of increase of the applied moment has on the critical buckling load. We find a delaying effect that is well described by a power law. In the closing Section 7 we argue that in addition to inertia, a gyroscopic effect may be responsible for this delay. We also discuss applications of our results and propose that they may help explain delayed torsional buckling observed in simulations of flexible marine risers [21].

\section{Cosserat rod formulation}

We describe the dynamic behaviour of slender structures by the Cosserat theory of thin rods. The rod is assumed to be inextensible, unshearable and intrinsically straight. For the background of the Cosserat theory the reader is referred to [1].

Let $\boldsymbol{r}(s, t):[0, L] \times \mathbb{R} \rightarrow \mathbb{R}^{3}$ be a smooth space curve of length $L$ describing the cen- 


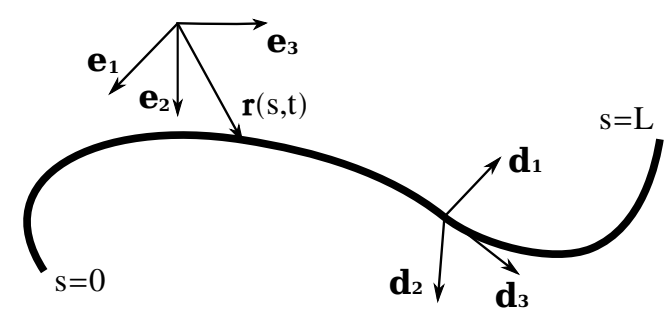

Figure 1: Cosserat model of a rod.

terline of the rod (see Fig 11). Here $s$ is arclength along the rod and $t$ is time. Further, let $\left\{\boldsymbol{d}_{1}(s, t), \boldsymbol{d}_{2}(s, t), \boldsymbol{d}_{3}(s, t)\right\}$ be a frame of orthonormal directors (the Cosserat triad) such that $\boldsymbol{d}_{1}$ and $\boldsymbol{d}_{2}$ span the cross-section of the rod at $s$, pointing along its principal bending axes, and $\boldsymbol{d}_{3}=\boldsymbol{d}_{1} \times \boldsymbol{d}_{2}$ is perpendicular to it, together forming the body frame to describe the orientation of each cross-section with respect to a fixed inertial frame $\left\{\boldsymbol{e}_{1}, \boldsymbol{e}_{2}, \boldsymbol{e}_{3}\right\}$. Orthonormality of the body frame implies the existence of two axial vectors $\boldsymbol{\kappa}$ (the curvature vector) and $\boldsymbol{\omega}$ (the spin vector) such that

$$
\partial_{s} \boldsymbol{d}_{k}=\boldsymbol{\kappa} \times \boldsymbol{d}_{k}, \quad \partial_{t} \boldsymbol{d}_{k}=\boldsymbol{\omega} \times \boldsymbol{d}_{k} \quad(k=1,2,3) .
$$

The equations of motion of a rod subjected to end loads only are

$$
\begin{aligned}
\partial_{s} \boldsymbol{n} & =\rho A \partial_{t t} \boldsymbol{r}, \\
\partial_{s} \boldsymbol{m}+\partial_{s} \boldsymbol{r} \times \boldsymbol{n} & =\rho \partial_{t}(\boldsymbol{I} \boldsymbol{\omega}),
\end{aligned}
$$

where $\boldsymbol{n}$ and $\boldsymbol{m}$ are the resultant contact force and contact couple acting at the centreline at $s$ [1]. $\boldsymbol{I}$ is the moment of inertia tensor, $A$ is the area of the cross-section and $\rho$ is the density.

Since the rod is inextensible and unshearable, the director $\boldsymbol{d}_{3}$ coincides with the tangent to the centreline of the rod, i.e., $\partial_{s} \boldsymbol{r}=\boldsymbol{d}_{3}$. If we also introduce the centreline velocity $\boldsymbol{u}=\partial_{t} \boldsymbol{r}$, then the identity $\partial_{t} \partial_{s} \boldsymbol{r}(s, t)=\partial_{s} \partial_{t} \boldsymbol{r}(s, t)$ can be written as

$$
\partial_{t} \boldsymbol{d}_{3}=\partial_{s} \boldsymbol{u} .
$$

Similarly, the identity $\partial_{t} \partial_{s} \boldsymbol{d}_{k}(s, t)=\partial_{s} \partial_{t} \boldsymbol{d}_{k}(s, t)$, on using Eq. (1), leads to the curvature-spin compatibility equation

$$
\partial_{s} \boldsymbol{\omega}=\partial_{t} \boldsymbol{\kappa}+\boldsymbol{\kappa} \times \boldsymbol{\omega} .
$$

When expressed in the body frame, Eqs (2), (3), (4), (5) give us the following equations (using a bold Roman font for triples of components in the body frame, writing $\mathbf{k}$ and $\mathbf{w}$ for the curvature and spin triples, respectively, and denoting $\boldsymbol{I}$ on principal axes by $\mathbf{I}=\operatorname{diag}\left(I_{1}, I_{2}, J\right)$ ):

$$
\begin{aligned}
\rho A \partial_{t} \mathbf{u} & =\partial_{s} \mathbf{n}+\mathbf{k} \times \mathbf{n}-\rho A(\mathbf{w} \times \mathbf{u}), \\
\rho \mathbf{I} \partial_{t} \mathbf{w} & =\partial_{s} \mathbf{m}+\mathbf{k} \times \mathbf{m}+\mathbf{d}_{3} \times \mathbf{n}-\rho(\mathbf{w} \times \mathbf{I} \mathbf{w}), \\
\partial_{t} \mathbf{k} & =\partial_{s} \mathbf{w}+\mathbf{k} \times \mathbf{w}, \\
\mathbf{0} & =\partial_{s} \mathbf{u}+\mathbf{k} \times \mathbf{u}-\mathbf{w} \times \mathbf{d}_{3} .
\end{aligned}
$$

We finally introduce linear constitutive relations between the curvatures $\mathbf{k}$ and the (body) moments $\mathbf{m}: \mathbf{m}=\hat{\mathbf{K}} \mathbf{k}$, where $\hat{\mathbf{K}}=\operatorname{diag}\left(B_{1}, B_{2}, C\right)$, with $B_{1}=E I_{1}$ and $B_{2}=E I_{2}$ the principal bending stiffnesses and $C=G J$ the torsional stiffness, $E$ and $G$ being Young's modulus and the 
shear modulus respectively. We note that for a homogeneous and isotropic material these elastic moduli are related by $G=E /[2(1+\nu)]$, where $\nu$ is Poisson's ratio. The constitutive relations can be used to eliminate $\mathbf{m}$ in Eq. (6) in favour of $\mathbf{k}$, thus giving us 12 equations for the body components $(\mathbf{u}, \mathbf{w}, \mathbf{k}, \mathbf{n})=: \boldsymbol{y}$ in the form

$$
\boldsymbol{M} \partial_{t} \boldsymbol{y}(s, t)+\boldsymbol{K} \partial_{s} \boldsymbol{y}(s, t)+\boldsymbol{F}(s, t)=\mathbf{0},
$$

where $M$ and $\boldsymbol{K}$ are mass and stiffness matrices (constant in the case of a uniform rod). All nonlinear terms in Eq. (6) are contained in the load vector $\boldsymbol{F}$. The same formulation is used in [11, 23].

\section{Generalised- $\alpha$ method}

The generalised- $\alpha$ method is a Newmark-like implicit integrator with desirable features such as second-order accuracy, unconditional stability and controllable numerical dissipation, first proposed by Chung and Hulbert in 1993 for the time integration of linear (second-order) structural dynamics problems [4]. The method was extended to first-order systems by Jansen et al. [14]. Erlicher at al. [5] verified the accuracy and stability of the method when applied to nonlinear dynamics problems. We apply the generalised $\alpha$-method for both spatial and temporal discretisation (of our first-order system Eq. (7)) as in [11, 23].

In the first step, we derive the semi-discrete form of Eq. (7) with respect to time as

$$
\left(\boldsymbol{M} \partial_{t} \boldsymbol{y}\right)^{1-\alpha_{t}}+\left(\boldsymbol{K} \partial_{s} \boldsymbol{y}\right)^{1-\beta_{t}}+\boldsymbol{F}^{1-\beta_{t}}(\boldsymbol{y})=\mathbf{0}
$$

where $(\cdot)^{1-\iota}=(1-\iota)(\cdot)^{i}+\iota(\cdot)^{i-1} \cdot \alpha_{t}$ and $\beta_{t}$ average the inertial term and terms of the stiffness and the load vector of the rod system, respectively, in time. $\boldsymbol{M}$ and $\boldsymbol{K}$ are constant in our case. As to the interpretation of the nonlinear $\boldsymbol{F}^{i}$ term, we choose the generalised trapezoidal rule (one of the three quadrature rules summarised in [5]), which corresponds to setting $\boldsymbol{F}^{1-\beta_{t}}(\boldsymbol{y})=(1-$ $\left.\beta_{t}\right) \boldsymbol{F}\left(\boldsymbol{y}^{i}\right)+\beta_{t} \boldsymbol{F}\left(\boldsymbol{y}^{i-1}\right)$. Eq. (8) is then expressed as

$$
\begin{aligned}
\boldsymbol{M}\left[\left(1-\alpha_{t}\right) \partial_{t} \boldsymbol{y}^{i}\right. & \left.+\alpha_{t} \partial_{t} \boldsymbol{y}^{i-1}\right] \\
& +\boldsymbol{K}\left[\left(1-\beta_{t}\right) \partial_{s} \boldsymbol{y}^{i}+\beta_{t} \partial_{s} \boldsymbol{y}^{i-1}\right] \\
& +\left(1-\beta_{t}\right) \boldsymbol{F}\left(\boldsymbol{y}^{i}\right)+\beta_{t} \boldsymbol{F}\left(\boldsymbol{y}^{i-1}\right)=\mathbf{0 .} .
\end{aligned}
$$

Similarly, applying the generalised- $\alpha$ method in space gives

$$
\begin{aligned}
\boldsymbol{M}\{ & \left.\left(1-\alpha_{t}\right)\left[\left(1-\alpha_{s}\right) \partial_{t} \boldsymbol{y}_{j}^{i}+\alpha_{s} \partial_{t} \boldsymbol{y}_{j-1}^{i}\right]+\alpha_{t}\left[\left(1-\alpha_{s}\right) \partial_{t} \boldsymbol{y}_{j}^{i-1}+\alpha_{s} \partial_{t} \boldsymbol{y}_{j-1}^{i-1}\right]\right\} \\
& +\boldsymbol{K}\left\{\left(1-\beta_{t}\right)\left[\left(1-\beta_{s}\right) \partial_{s} \boldsymbol{y}_{j}^{i}+\beta_{s} \partial_{s} \boldsymbol{y}_{j-1}^{i}\right]+\beta_{t}\left[\left(1-\beta_{s}\right) \partial_{s} \boldsymbol{y}_{j}^{i-1}+\beta_{s} \partial_{s} \boldsymbol{y}_{j-1}^{i-1}\right]\right\} \\
& +\left\{\left(1-\beta_{t}\right)\left[\left(1-\beta_{s}\right) \boldsymbol{F}_{j}^{i}+\beta_{s} \boldsymbol{F}_{j-1}^{i}\right]+\beta_{t}\left[\left(1-\beta_{s}\right) \boldsymbol{F}_{j}^{i-1}+\beta_{s} \boldsymbol{F}_{j-1}^{i-1}\right]\right\}=\mathbf{0} .
\end{aligned}
$$

In this equation the superscript indicates the time step while the subscript indicates the spatial step, i.e., the node of the discretised curve.

In the second step the $s$ and $t$ derivatives are approximated as

$$
\partial_{t} \boldsymbol{y}^{i}=\frac{\boldsymbol{y}^{i}-\boldsymbol{y}^{i-1}}{\gamma_{t} \Delta t}-\frac{1-\gamma_{t}}{\gamma_{t}} \partial_{t} \boldsymbol{y}^{i-1}, \quad \partial_{s} \boldsymbol{y}_{j}=\frac{\boldsymbol{y}_{j}-\boldsymbol{y}_{j-1}}{\gamma_{s} \Delta s}-\frac{1-\gamma_{s}}{\gamma_{s}} \partial_{s} \boldsymbol{y}_{j-1},
$$


where $\Delta s$ and $\Delta t$ are the spatial step size and time step size, respectively. By substituting Eq. (11) into Eq. (10) we obtain a system of algebraic equations in the form

$$
\boldsymbol{\Lambda}_{j}^{i}:=\boldsymbol{A}\left(\boldsymbol{y}_{j}^{i}\right)+\boldsymbol{B}\left(\boldsymbol{y}_{j-1}^{i}\right)-\zeta \partial_{s}(\boldsymbol{K} \boldsymbol{y})_{j-1}^{i}-\boldsymbol{R}_{j-1}^{i-1}=\mathbf{0}
$$

with

$$
\zeta=\frac{\left(1-\beta_{t}\right)\left(1-\beta_{s}-\gamma_{s}\right)}{\gamma_{s}}
$$

Here $\boldsymbol{A}\left(\boldsymbol{y}_{j}^{i}\right)$ and $\boldsymbol{B}\left(\boldsymbol{y}_{j-1}^{i}\right)$ are nonlinear functions of the unknown variables, while $\boldsymbol{R}_{j-1}^{i-1}:=$ $\boldsymbol{R}_{j-1}^{i-1}\left(\boldsymbol{y}_{j}^{i-1}, \boldsymbol{y}_{j-1}^{i-1}, \partial_{t} \boldsymbol{y}_{j}^{i-1}, \partial_{t} \boldsymbol{y}_{j-1}^{i-1}, \partial_{s} \boldsymbol{y}_{j-1}^{i-1}\right)$ is known once variables at the earlier timestep $\boldsymbol{y}^{i-1}$ are solved. Each set of three parameters $\alpha_{s}, \beta_{s}, \gamma_{s}$ and $\alpha_{t}, \beta_{t}, \gamma_{t}$ can be expressed as functions of $\lambda^{\infty}$, the eigenvalue of the amplification matrix at infinity [4]:

$$
\alpha_{(\cdot)}=\frac{3 \lambda_{(\cdot)}^{\infty}+1}{2 \lambda_{(\cdot)}^{\infty}-2}, \quad \beta_{(\cdot)}=\frac{\lambda_{(\cdot)}^{\infty}}{\lambda_{(\cdot)}^{\infty}-1}, \quad \gamma_{(\cdot)}=\frac{1}{1-\lambda_{(\cdot)}^{\infty},}
$$

where $\lambda_{(\cdot)}^{\infty} \in[-1,0]$. Note that then $\beta_{(\cdot)}+\gamma_{(\cdot)}=1$ and hence

$$
\zeta=0 .
$$

This allows us to eliminate the terms with $\zeta$ in Eq. (12) thereby significantly simplifying the Jacobian matrix $\partial \boldsymbol{\Lambda}_{j}^{i} / \partial \boldsymbol{y}_{l}^{k}$ of the nonlinear system of equations without loss of either accuracy or numerical stability. We finally need to specify $\lambda_{(\cdot)}^{\infty}$. We choose $\lambda_{s}^{\infty}=\lambda_{t}^{\infty}=-1$, corresponding to a value of $1 / 2$ for all six parameters, which gives a stable second-order scheme without numerical damping. The final system of algebraic Eqs. (12) is solved with the global Newton code NLEQ1 [19].

\section{Computing rod shapes}

Since we solve the rod formulations in the body frame instead of the fixed inertial frame, to compute rod shapes in space we need to postprocess the solution $\boldsymbol{y}$ by subsequently solving either of the equations in Eq. (1) and integrating the centreline equation $\partial_{s} \boldsymbol{r}=\boldsymbol{d}_{3}$ or the velocity $\boldsymbol{u}=\partial_{t} \boldsymbol{r}$ to obtain $\boldsymbol{r}$. We define $\boldsymbol{R}$ as the matrix whose columns are the frame vectors $\boldsymbol{e}_{1}, \boldsymbol{e}_{2}, \boldsymbol{e}_{3}$ represented in the body frame. $\boldsymbol{R}$ is thus the rotation matrix from the inertial to the body frame, satisfying

$$
\boldsymbol{e}_{k}=\boldsymbol{R} \boldsymbol{d}_{k} \quad(k=1,2,3) .
$$

For any vector $\boldsymbol{V}$ we have the following transformation rule for derivatives with respect to the different frames:

$$
\left.\partial_{s} \boldsymbol{V}\right|_{\left\{\boldsymbol{e}_{k}\right\}}=\left.\partial_{s} \mathbf{V}\right|_{\left\{\boldsymbol{d}_{k}\right\}}+\mathbf{k} \times \mathbf{V},\left.\quad \partial_{t} \boldsymbol{V}\right|_{\left\{\boldsymbol{e}_{k}\right\}}=\left.\partial_{t} \mathbf{V}\right|_{\left\{\boldsymbol{d}_{k}\right\}}+\mathbf{w} \times \mathbf{V} \quad(k=1,2,3) .
$$

Applying these to the inertial frame vectors, we have

$$
\partial_{s} \mathbf{e}_{k}=-\mathbf{k} \times \mathbf{e}_{k}=-\widehat{\mathbf{k}} \mathbf{e}_{k}, \quad \partial_{t} \mathbf{e}_{k}=-\mathbf{w} \times \mathbf{e}_{k}=-\widehat{\mathbf{w}} \mathbf{e}_{k},
$$

where $\widehat{\mathbf{k}}$ and $\widehat{\mathbf{w}}$ are the skew-symmetric matrices corresponding to $\mathbf{k}$ and $\mathbf{w}$. It follows that

$$
\partial_{s} \boldsymbol{R}=-\widehat{\mathbf{k}} \boldsymbol{R}, \quad \partial_{t} \boldsymbol{R}=-\widehat{\mathbf{w}} \boldsymbol{R} .
$$




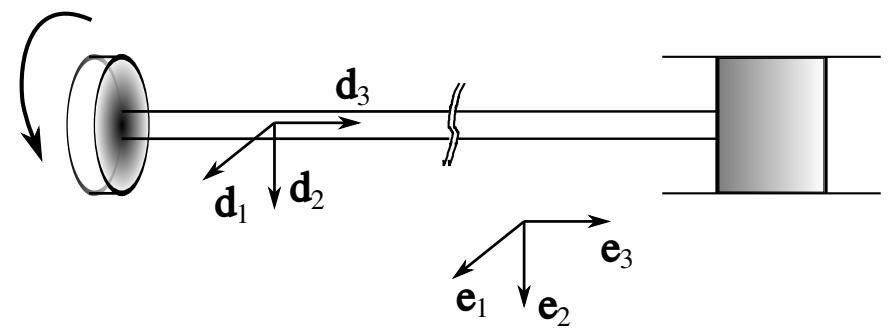

Figure 2: Sketch of a straight rod under torsion.

Assuming we choose to use the second equation in Eq. (17) (i.e., time integration), the rotation matrix is incrementally updated according to

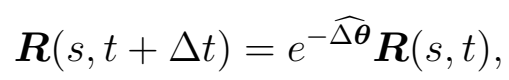

with initial condition $\boldsymbol{R}(0)=\boldsymbol{E}$ (the identity matrix) if the body frame at $t=0$ is aligned with the fixed frame [18]. Here, $\widehat{\Delta \boldsymbol{\theta}}$ is the skew-symmetric matrix of the incremental rotation vector $\Delta \boldsymbol{\theta}$ with respect to the spin vector $\mathbf{w}$, where

$$
\Delta \boldsymbol{\theta}=\frac{\mathbf{w}(s, t+\Delta t)+\mathbf{w}(s, t)}{2} \Delta t .
$$

The exponential is conveniently computed by using the Rodrigues formula (valid for any skewsymmetric matrix $\widehat{\mathbf{x}}$ )

$$
e^{-\widehat{\mathbf{x}}}=\boldsymbol{E}-\widehat{\mathbf{n}} \sin |\mathbf{x}|+\widehat{\mathbf{n}}^{2}(1-\cos |\mathbf{x}|), \quad \mathbf{n}=\frac{\mathbf{x}}{|\mathbf{x}|} .
$$

Having found $\boldsymbol{R}$, we finally obtain the centreline $\boldsymbol{r}$ by integrating the velocity vector:

$$
\partial_{t} \boldsymbol{r}=\boldsymbol{u}=\boldsymbol{R}^{\mathrm{T}} \mathbf{u}
$$

\section{Prebuckling torsional waves}

The unstressed rod is taken to be straight and to lie along the basis vector $\boldsymbol{e}_{3}$ of the fixed inertial frame. The left end of the rod is fixed in position and subjected to a twisting moment while the right end is constrained in the $e_{1}$ and $\boldsymbol{e}_{2}$ directions and allowed to move in the $\boldsymbol{e}_{3}$ direction, as illustrated in Fig. 2 .

If the twisting moment varies in time then a torsional wave will propagate along the straight centreline before the rod buckles. In this situation $\kappa_{3}$ and $\omega_{3}$ are the only two nonzero variables in the rod. For notational simplicity we shall set $\kappa:=\kappa_{3}$ and $\omega:=\omega_{3}$ in this section. The governing wave equation can be written with respect to either $\kappa$ or $\omega$, by setting all other variables to zero in 
Eq. (6) and combining the reduced moment balance equation $\rho J \partial_{t} \omega=G J \partial_{s} \kappa$ with the director compatibility equation $\partial_{t} \kappa=\partial_{s} \omega$ :

$$
\partial_{t t} \kappa=c^{2} \partial_{s s} \kappa \quad \text { or } \quad \partial_{t t} \omega=c^{2} \partial_{s s} \omega
$$

where $c=\sqrt{\frac{G}{\rho}}$ is the wave speed. We shall formulate an initial-boundary-value problem in terms of the variable $\kappa$. The initial condition (IC) $\omega(s, 0)=0$ can be written as $\partial_{s} \omega(s, 0)=0$, and thus $\partial_{t} \kappa(s, 0)=0$, by applying the reduced director compatibility equation. Similarily, the boundary condition $(\mathrm{BC}) \omega(L, t)=0$ can be written as $\partial_{s} \kappa(L, t)=0$ by applying the reduced moment balance equation. The torsional rod experiment is thus governed by

$$
\begin{aligned}
& \partial_{t t} \kappa=c^{2} \partial_{s s} \kappa, \\
& \text { IC: } \quad \kappa(s, 0)=h(0), \quad \partial_{t} \kappa(s, 0)=0 \text {, } \\
& \text { BC: } \quad \kappa(0, t)=h(t), \quad \partial_{s} \kappa(L, t)=0 \text {, }
\end{aligned}
$$

where $h(t)$ is the twist function applied at the left end while $h(0)$ is its initial value.

Using Duhamel's principle, we find the analytical solution for Eq. (23) as follows:

$$
\kappa(s, t)=h(t)+\sum_{n=1}^{\infty} A_{n} \sin c \sqrt{\lambda_{n}} t \sin \sqrt{\lambda_{n}} s+\int_{0}^{t} \eta(s, t ; \tau) \mathrm{d} \tau,
$$

where

$$
\begin{aligned}
& A_{n}=-\frac{2}{L c \sqrt{\lambda_{n}}} \int_{0}^{L} h^{\prime}(0) \sin \sqrt{\lambda_{n}} s \mathrm{~d} s=-\frac{2 h^{\prime}(0)}{L c \lambda_{n}}, \\
& \eta(s, t ; \tau)=-\sum_{n=1}^{\infty} \frac{2}{L c \lambda_{n}} h^{\prime \prime}(\tau) \sin c \sqrt{\lambda_{n}}(t-\tau) \sin \sqrt{\lambda_{n}} s
\end{aligned}
$$

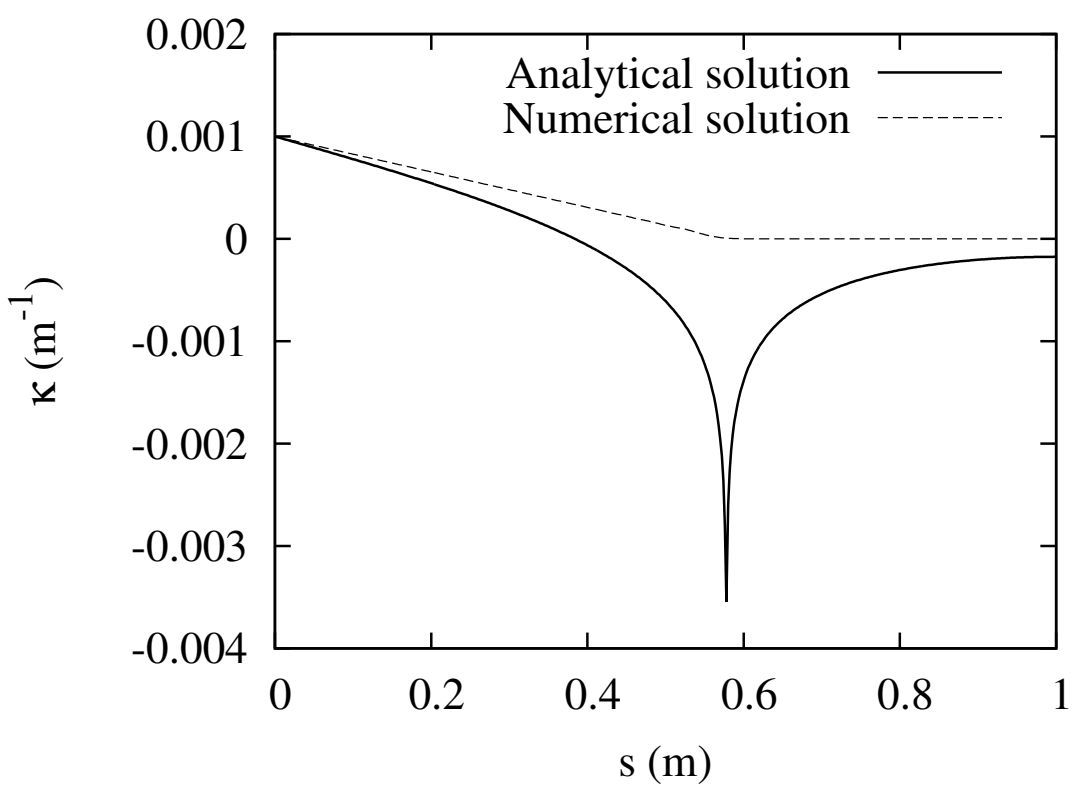

Figure 3: Torsional wave under linear loading $(c=57.74 \mathrm{~m} / \mathrm{s}, t=0.01 \mathrm{~s}, \Delta s=0.002 \mathrm{~m}, \Delta t=$ $0.001 \mathrm{~s})$. 
and eigenvalues $\sqrt{\lambda_{n}}=\frac{(2 n-1) \pi}{2 L}(n=1,2, \cdots)$. If we take a linearly increasing function for the twist boundary condition, i.e., $h(t)=\kappa_{0}+a t$, the analytical solution (24) becomes

$$
\kappa(s, t)=\kappa_{0}+a t-\sum_{n=1}^{\infty} \frac{2 a}{L c \lambda_{n}} \sin c \sqrt{\lambda_{n}} t \sin \sqrt{\lambda_{n}} s,
$$

where $\kappa_{0}$ is the initial twist and $a$ is the loading speed (i.e., the rod is pretwisted by a twisting moment $C h(0)$ and the end moment is then varied according to $C h(t)$ ).

The analytical solution (for $L=1 \mathrm{~m}, c=57.74 \mathrm{~m} / \mathrm{s}, \kappa_{0}=0 \mathrm{~m}^{-1}, a=0.1 \mathrm{rad} / \mathrm{s}$ and at time $t=0.01 \mathrm{~s}$ ) is displayed in Fig. 3. Here we have truncated the series in Eq. (25) after the first 1000 terms (keeping more terms does not alter the plot) and taken $\Delta s=0.002 \mathrm{~m}$ to resolve the sharp peak.

The sharp peak in $\kappa$ is a result of the incompatibility between boundary and initial conditions in Eq. (23) (if the initial conditions are to hold for all $s \in[0, L]$ and the boundary conditions for all $t \geq 0$ ). Note that this incompatibility is entirely physical: by applying a twisting moment $\kappa$ that varies in time (however slowly the change), one automatically applies an angular velocity $\omega$ as well, which in turn, because of the relationships between $\kappa$ and $\omega$ leads to higher-order derivatives of $\kappa$, etc. Specifically, in this case with $h(t)=\kappa_{0}+a t$, we have $\partial_{t} \kappa(0, t)=h^{\prime}(t)=a$, which disagrees with the second initial condition in Eq. (23) at $t=0$ unless $a=0$. The solution is thus discontinuous at $t=0$. And had we chosen $h(t)=\kappa_{0}+a t^{2}$ then we could have taken one more derivative and used the equation $\partial_{t t} \kappa=c^{2} \partial_{s s} \kappa$ to reach a disagreement with the (twice differentiated) first initial condition in Eq. (23) at $t=0$ (unless $a=0$ ). A discontinuity can only be avoided by having infinitely slow speed-up $h$, i.e., by having end velocity $\partial_{t} \kappa(0, t)$, acceleration $\partial_{t t} \kappa(0, t)$ and all higher-order derivatives equal to zero at $t=0$. As commented in [3, 6], the initial and boundary conditions of a PDE need to be infinitely compatible to avoid singularities in the solution at corners of the space-time domain. But this is a problem for our numerical scheme, which is not designed to approximate discontinuous solutions, and hence the discrepancy between analytical and numerical solution, computed using the generalised- $\alpha$ method described in the previous section, seen in Fig. 3 .

To look more closely into this singularity phenomenon, we take a twist-release example in which the rod is initially twisted and instantaneously released at $t=0$. This experiment is described by

$$
\begin{array}{cc}
\partial_{t t} \kappa=c^{2} \partial_{s s} \kappa, \\
\text { IC: } \quad \kappa(s, 0)=\kappa_{0}, \quad \partial_{t} \kappa(s, 0)=0, \\
\text { BC: } \quad \partial_{s} \kappa(0, t)=0, \quad \kappa(L, t)=0,
\end{array}
$$

with analytical solution

$$
\kappa(s, t)=\sum_{n=1}^{\infty} \frac{2 \kappa_{0}}{L \sqrt{\lambda_{n}}}(-1)^{n-1} \cos c \sqrt{\lambda_{n}} t \cos \sqrt{\lambda_{n}} s
$$

and eigenvalues $\sqrt{\lambda_{n}}=\frac{(2 n-1) \pi}{2 L}(n=1,2, \cdots)$. Clearly, the initial condition $\kappa(s, 0)$ is not compatible with the boundary condition $\kappa(L, t)$. Note that, for this experiment, the analytical solution (for $t>0$ ) is a travelling square wave with maximum value $\kappa_{0}$ and minimum value 0 . Fig. 4 shows that the numerical code cannot capture the torsional wave in this block shape $\left(\kappa_{0}=10 \mathrm{~m}^{-1}\right)$.

In a real experiment, the twist is never released instantaneously but within a finite time. To model this we use the infinitely smooth transition function $f$ (taken from [13]) shown in Fig. 5. 


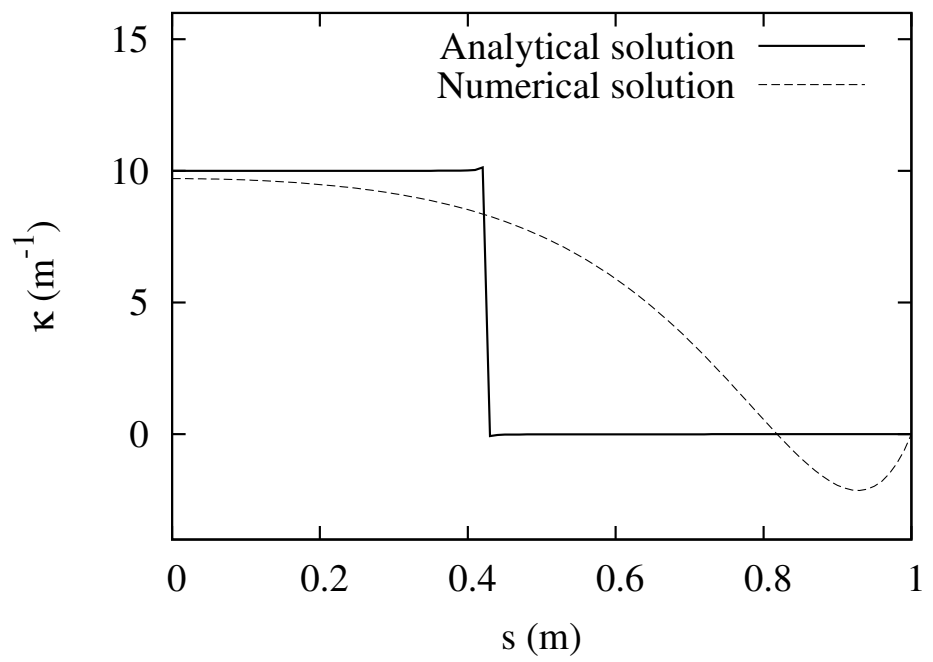

Figure 4: Torsional wave under instantaneous release $(c=57.74 \mathrm{~m} / \mathrm{s}, t=0.01 \mathrm{~s})$.

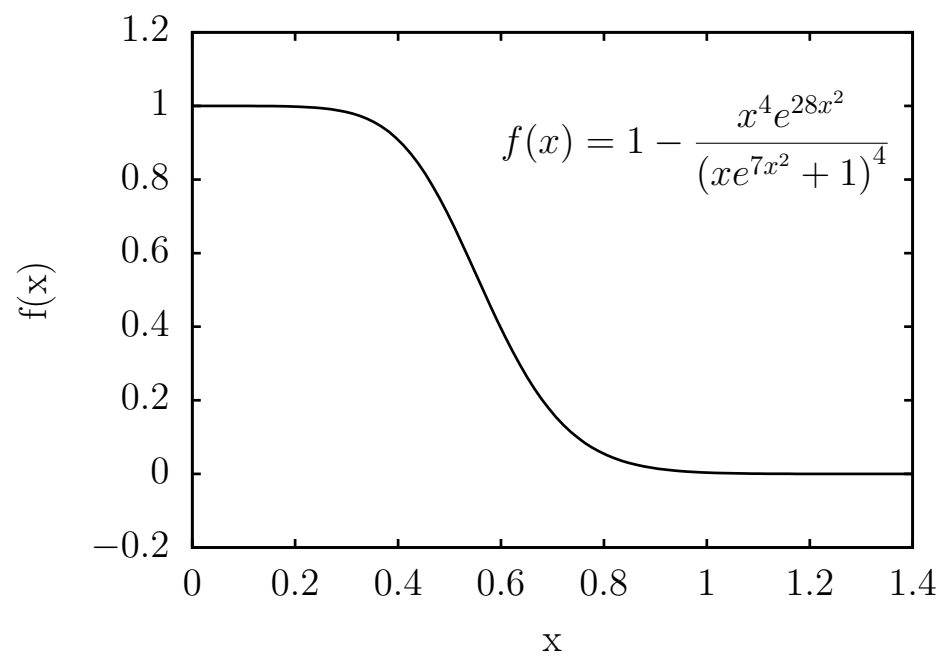

Figure 5: An infinitely smooth function $f(x)$. 


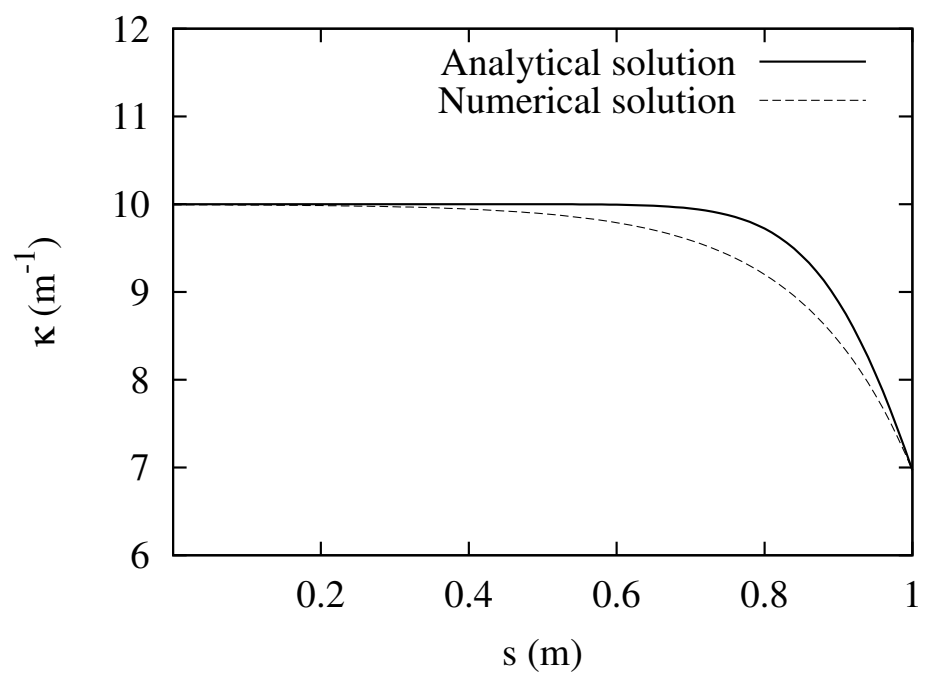

Figure 6: Torsional wave under smooth release $(c=57.74 \mathrm{~m} / \mathrm{s}, t=0.01 \mathrm{~s})$.

The dynamic boundary condition of the torsional wave is made infinitely smooth by replacing the right-end boundary condition in Eq. (26) by $\kappa_{0} f(50 t)$, describing a release of the twist within approximately $0.02 \mathrm{~s}$. The new set of equations is thus

$$
\begin{array}{ll} 
& \partial_{t t} \kappa=c^{2} \partial_{s s} \kappa, \\
\text { IC: } & \kappa(s, 0)=\kappa_{0}, \quad \partial_{t} \kappa(s, 0)=0, \\
\text { BC: } & \partial_{s} \kappa(0, t)=0, \quad \kappa(L, t)=\kappa_{0} f(50 t),
\end{array}
$$

with analytical solution

$$
\kappa(s, t)=\kappa_{0} f(50 t)+\int_{0}^{t} \eta(s, t ; \tau) \mathrm{d} \tau,
$$

where

$$
\eta(s, t ; \tau)=\sum_{n=1}^{\infty}(-1)^{n} \frac{5000}{L c \lambda_{n}} \kappa_{0} f^{\prime \prime}(50 \tau) \sin c \sqrt{\lambda_{n}}(t-\tau) \cos \sqrt{\lambda_{n}} s
$$

and eigenvalues $\sqrt{\lambda_{n}}=\frac{(2 n-1) \pi}{2 L}(n=1,2, \cdots)$. We get good agreement between analytical solution and numerical solution, as seen in Fig. 6, by only taking the first 100 terms in Eq. 29) for the anaytical solution and $\Delta t=0.005 \mathrm{~s}$ for the numerical solution. For both, $\Delta s=0.01 \mathrm{~m}$. This suggests that taking smoothing functions is a good (as well as realistic) way to resolve the compatibility problem of discontinuous waves. The remaining difference in Fig. 6 is probably due to the fact that although the twist release is infinitely smooth, it is not infinitely slow at $t=0$. As a consequence, high-order compatibility conditions are not satisfied because, while $f^{\prime}(0)=f^{\prime \prime}(0)=f^{\prime \prime \prime}(0)=0$, we have $f^{\prime \prime \prime \prime}(0)=-24$, and hence $\partial_{t t t t} \kappa(L, t)=(50)^{4} \kappa_{0} f^{\prime \prime \prime \prime \prime}(50 t)$, implying $\partial_{t t t t} \kappa(L, 0) \neq 0$, which is incompatible with $0=c^{4} \partial_{s s s s} \kappa(s, 0)=c^{2} \partial_{s s} \partial_{t t} \kappa(s, 0)=$ $c^{2} \partial_{t t} \partial_{s s} \kappa(s, 0)=\partial_{t t t t} \kappa(s, 0)$. 


\section{Torsional buckling}

For the dynamic buckling study we take a rod of circular cross-section with the physical parameters listed in Table 1. The twist load is linearly increased at the left end. We have the following boundary conditions:

$$
\begin{array}{rrrr}
s=0: & \mathbf{u}=\mathbf{0}, & \omega_{1}=\omega_{2}=0, & \kappa_{3}=\kappa_{0}+a t, \\
s=L: & \mathbf{w}=\mathbf{0}, & \mathrm{u}_{1}=\mathrm{u}_{2}=0, & \mathrm{n}_{3}=0 .
\end{array}
$$

The initial condition for an unstressed straight and prismatic rod is $\boldsymbol{y}(s, 0)=\boldsymbol{0}$. We solve for $\boldsymbol{y}(s, t)$ first and then integrate rod shapes $\boldsymbol{r}(s, t)$ by the postprocessing procedure discussed in Section 4. To observe the buckling phenomenon (rather than computing an unstable solution), a small perturbative force, of magnitude $10^{-8} \mathrm{~N}$, is added in the $\boldsymbol{d}_{1}$ direction.

Fig. 7 shows five representative snapshots during the dynamic evolution of torsional buckling with $\kappa_{0}=0 \mathrm{~m}^{-1}$ and $a=1.6 \mathrm{rad} / \mathrm{s}$. The rod initially takes an approximately helical shape, with gradually increasing radius, which subsequently localises, in agreement with the torsional buckling studies in [24] and [8]. The shape is more localised at the right end due to the asymmetry of the dynamical boundary conditions (one end is twisted while the other end is not). Without a repulsive force in the physical model, the rod will eventually pass through itself when the shape gets more localised.

In order to detect the onset of buckling we measure the variation of the axial velocity $u_{3}$ at the free (right) end of the rod: $u_{3}$ equals zero as long as the rod is straight and starts to vary dramatically when the rod starts to buckle, as visualised in Fig. 7. Linearly fitting the initial dramatic variation and extrapolating back to $\mathrm{u}_{3}=0$ gives an estimate for the critical time of buckling and hence the critical twist $\kappa_{3}$, as demonstrated in Fig. 8 and Fig. 9 (for different values of the density $\rho$ ). We conclude from these plots that the dynamical critical load is larger for rods of larger density, which seems consistent with the larger inertia of such rods.

The critical buckling load in a quasi-static analysis is [26]

$$
\mathrm{M}_{\mathrm{cri}}=G J \kappa_{\mathrm{cri}}=\frac{2 \pi E I_{1}}{L} \mathrm{~m}_{\mathrm{cri}}
$$

where $\mathrm{m}_{\text {cri }}=1.4303$ is the nondimensional critical twisting moment (the smallest nonzero root of $\tan (\pi \mathrm{m})=\pi \mathrm{m})$. For a rod with the physical properties listed in Table 1 the quasi-static critical

Table 1: Physical parameters of the example circular rod.

\begin{tabular}{lll}
\hline Parameter & Unit (SI) & Value \\
\hline Young's modulus $E$ & $\mathrm{~Pa}$ & $1.25 \times 10^{7}$ \\
Poisson's ratio $\nu$ & - & 0.25 \\
Shear modulus $G$ & $\mathrm{~Pa}$ & $G=\frac{E}{2(1+\nu)}$ \\
Diameter $D$ & $\mathrm{~m}$ & 0.001 \\
Length $L$ & $\mathrm{~m}$ & 1 \\
Cross-sectional area $A$ & $\mathrm{~m}^{2}$ & $A=\frac{\pi D^{2}}{4}$ \\
Density $\rho$ & $\mathrm{kg} / \mathrm{m}^{3}$ & 1500 \\
Second moments of area (bending) & $\mathrm{m}^{4}$ & $I_{1,2}=\frac{A D^{2}}{16}$ \\
Second moment of area (twist) & $\mathrm{m}^{4}$ & $J=\frac{A D^{2}}{8}$ \\
\hline
\end{tabular}


(a)

(b)

(c)

(d)

(e) $\mathrm{t}=20.0 \mathrm{~s}, \kappa_{3}=32.0 \mathrm{~m}^{-1}$

$\mathrm{t}=21.0 \mathrm{~s}, \kappa_{3}=33.6 \mathrm{~m}^{-1}$

$\mathrm{t}=22.0 \mathrm{~s}, \kappa_{3}=35.2 \mathrm{~m}^{-1}$

$\mathrm{t}=22.5 \mathrm{~s}, \kappa_{3}=36.0 \mathrm{~m}^{-1}$

$\mathrm{t}=23.0 \mathrm{~s}, \kappa_{3}=36.8 \mathrm{~m}^{-1}$

Figure 7: Snapshots at selected times during the dynamic evolution of buckling: (a) prebuckling straight shape, (b) approximately helical shape, (c) helical shape with increased radius, (d) localised shape, (e) right before the rod self-contacts.

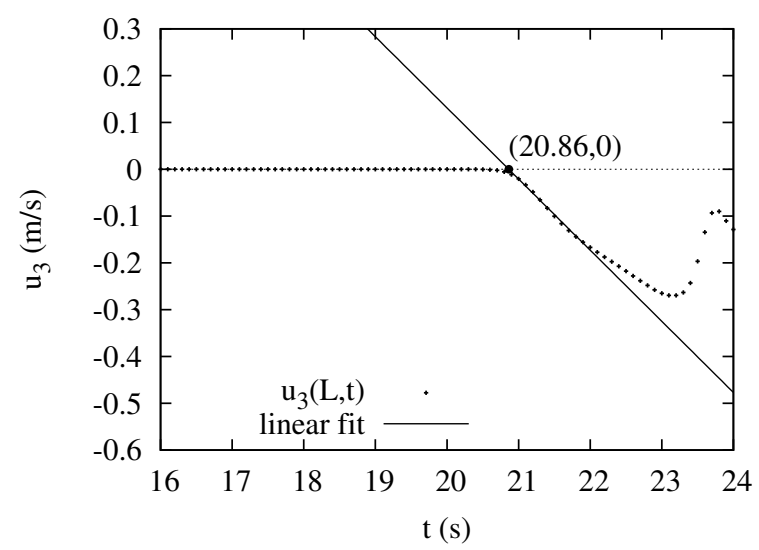

Figure 8: Axial velocity $\mathrm{u}_{3}$ at $s=L(\rho=$ $\left.1500 \mathrm{~kg} \cdot \mathrm{m}^{3}, \kappa_{0}=0 \mathrm{~m}^{-1}, a=1.6 \mathrm{rad} / \mathrm{s}\right)$

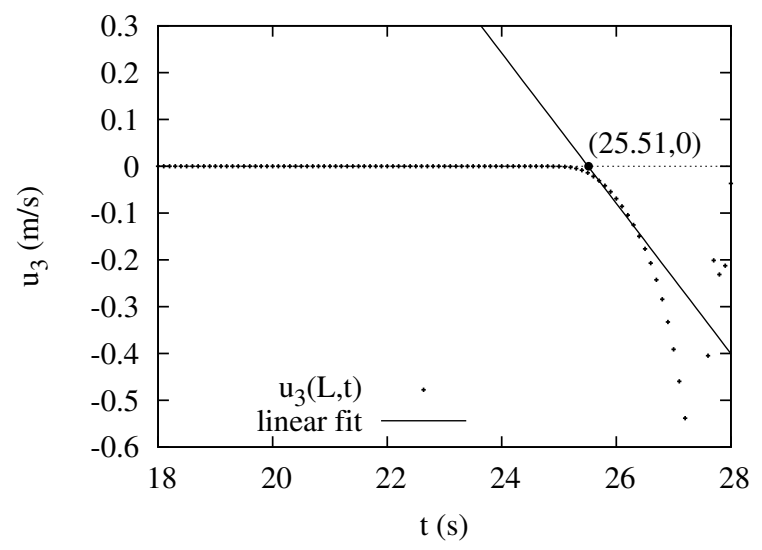

Figure 9: Axial velocity $\mathrm{u}_{3}$ at $s=L(\rho=$ $\left.5000 \mathrm{~kg} \cdot \mathrm{m}^{3}, \kappa_{0}=0 \mathrm{~m}^{-1}, a=1.6 \mathrm{rad} / \mathrm{s}\right)$ 
twist is

$$
\kappa_{\mathrm{cri}}=\frac{\mathrm{M}_{\mathrm{cri}}}{G J}=\frac{2 \pi E I_{1}}{L G J} \mathrm{~m}_{\mathrm{cri}}=11.2335 \mathrm{~m}^{-1} .
$$

We notice that the dynamic critical load $\kappa_{3}=20.86 a=33.38 \mathrm{~m}^{-1}$ found by the linear fit in Fig. 8 is much larger than the statics value 11.2335 . To quantify the effect of the loading speed $a$ on the critical twist we use the extrapolation method described above for different values of $a$. The result is shown in Fig. 10 (note that we have here taken a nonzero $\kappa_{0}$ value to avoid very long runs for small values of $a$ ). Extrapolation to the static buckling case ( $a=0$, dashed line in Fig. 10) gives good agreement with the value in Eq. 32. Fig. 10 quantifies the delay effect on torsional buckling due to the loading speed. The effect is seen to increase less than linearly with $a$. A least-squares fit to the simple power law

$$
\kappa_{3}=\kappa_{\mathrm{cri}}+\frac{b}{L}\left(\frac{a L}{c}\right)^{\sigma}
$$

gives

$$
b=160.60, \quad \sigma=0.5680 .
$$

This law predicts a 'time until buckling' of $t=20.11 \mathrm{~s}$ in Fig. 8 and $t=25.45 \mathrm{~s}$ in Fig. 9, in good agreement with the results in those figures.

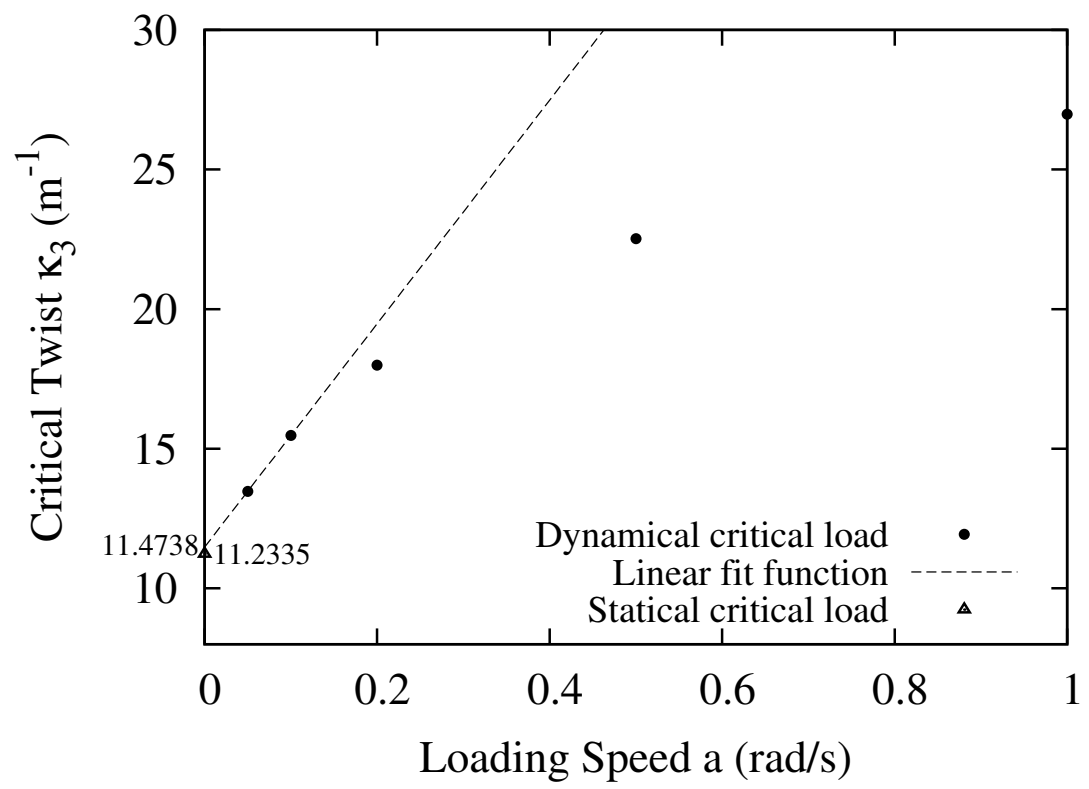

Figure 10: Delay effect on torsional critical twist $\kappa_{3}$ due to loading speed $a\left(\kappa_{0}=10 \mathrm{~m}^{-1}\right.$ and physical parameters are as listed in Table 1).

\section{Conclusion}

In this paper we have studied dynamic torsional buckling of a rod under an increasing end twisting moment, accounting for the finite time of application of the moment. The dynamic loading induces an incompatibility between initial and boundary conditions leading to discontinuous wave patterns that are hard to capture by the numerical solution method. In transient problems (e.g., the heat 
equation) or in problems with dissipation these discontinuities are smeared out over time, but this is not the case for our undamped wave equation. To deal with these incompatibilities we have applied a smoothing function. We argue that the modified boundary-value problem, describing twist release in finite time, is also a more accurate model of actual experiments in which twisting moments are never applied or released instantaneously.

Torsional buckling is observed when a critical amount of twist has been inserted into the rod. We find this critical amount of twist to be larger than the static critical twist, i.e., dynamic application of the twist leads to a delay of torsional buckling. A plausible explanation for the delay effect is inertia: it takes time for the unstable straight configuration to evolve into a stable bent configuration. Looked at it in another way: there is a constant trade between twist $\left(\kappa_{3}\right)$ and angular velocity $\left(\omega_{3}\right)$, so the actual moment in the rod constantly fluctuates. However, the period of torsional waves for the parameters in Section $6\left(T=2 \pi /\left(c \sqrt{\lambda_{1}}\right)=4 L / c=0.06928 \mathrm{~s}\right.$ for the first vibration mode) is much smaller than the delay found, which suggests that inertia does not provide the whole explanation. We suggest that gyroscopic stabilisation as a result of the angular velocity also plays a role.

Our results have various applications. Prebuckling torque oscillations are a major concern in (gas, steam or wind) turbine generators [12, 20, 27] where large transient torques induced by the fast reclosing of transmission lines may cause shaft fatigue damage or instability. In [20] an 18-mass model is discussed for the turbine shaft, expected to be capable of representing the machine's most significant torsional modes. A continuum model like ours would also be able to detect unexpectedly high modes. As to the effect of the rate of application of the end torque on torsional buckling, numerical simulations in [21] reveal a delay in torsional buckling (kink formation) of flexible marine risers being subjected to a torque during installation. The authors relate the delay to the frictional torque at the touch-down point. Our results suggest that even in the absence of such a torque a delay of torsional buckling might be expected.

\section{Acknowledgements}

XWZ gratefully acknowledges support from the China Scholarship Council.

\section{References}

[1] Antman SS. Nonlinear problems of elasticity (2nd ed.). New York: Springer-Verlag; 2005.

[2] Atanackovic TM, Glavardanov VB. Buckling of a twisted and compressed rod. Int J Solids Struct 2002;39:2987-2999.

[3] Boyd JP, Flyer N. Compatibility conditions for time-dependent partial differential equations and the rate of convergence of Chebyshev and Fourier spectral methods. Comput Methods Appl Mech Engrg 1999;175:281-309.

[4] Chung J, Hulbert GM. A time integration algorithm for structural dynamics with improved numerical dissipation - the generalized- $\alpha$ method. J Appl Mech 1993;60:371-375.

[5] Erlicher S, Bonaventura L, Bursi OS. The analysis of the generalized- $\alpha$ method for non-linear dynamic problems. Computational Mechanics 2002;28:83-104. 
[6] Flyer N, Swarztrauber PN. The convergence of spectral and finite difference methods for initial-boundary value problems. SIAM J Sci Comput 2002;23:1731-1751.

[7] Glavardanov V, Maretic RB. Stability of a twisted and compressed clamped rod. Acta Mech 2009;202:17-33.

[8] Goriely A, Tabor M. Nonlinear dynamics of filaments I. Dynamical instabilities. Physica D 1997;105:20-44.

[9] Goss VGA, van der Heijden GHM, Thompson JMT, Neukirch S. Experiments on snap buckling, hysteresis and loop formation in twisted rods. Exp Mech 2005;45:101-111.

[10] Goyal S, Perkins NC, Lee CL. Nonlinear dynamics and loop formation in Kirchhoff rods with implications to the mechanics of DNA and cables. J Comput Phys 2005;209:371-389.

[11] Goyal S, Perkins NC, Lee CL. Non-linear dynamic intertwining of rods with self-contact. Int J Non-Lin Mech 2008;43:65-73.

[12] Hammons TJ, Chanal L. Measurement of torque in steam turbine-generator shafts following severe disturbances on the electrical supply system. IEEE Transactions on Energy Conversion 1991;6:193-200.

[13] Hoffman KA, Manning RS. An extended conjugate point theory with application to the stability of planar buckling of an elastic rod subject to a repulsive self-potential. SIAM J Math Anal 2009;41:465-494.

[14] Jansen KE, Whiting CH, Hulbert GM. A generalized- $\alpha$ method for integrating the filtered Navier-Stokes equations with a stabilized finite element method. Comput Methods Appl Mech Engrg 2000;190:305-319.

[15] Ji W, Waas AM. Dynamic bifurcation buckling of an impacted column. Int J Eng Sci 2008;46:958-967.

[16] Klapper I. Biological applications of the dynamics of twisted elastic rods. J Comput Phys 1996;125:325-337.

[17] Kuzkin VA, Dannert MM. Buckling of a column under a constant speed compression: a dynamic correction to the Euler formula. Acta Mech 2016;227:1645-1652.

[18] Murray RM, Li ZX, Sastry SS. A mathematical introduction to robotic manipulation. Boca Raton: CRC Press; 1994.

[19] Nowak U, Weimann L. A family of Newton codes for systems of highly nonlinear equations. Technical Report TR-91-10. Konrad-Zuse-Zentrum für Informationstechnik Berlin; 1991.

[20] Ramos AJP, Mota WS, Dantas YS. Evaluation of torsional efforts on thermal machines shaft with gas turbine resulting of automatic reclosing. International Journal of Mechanical and Mechatronics Engineering 2007;1:497-502.

[21] Saevik S, Koloshkin E. Torsion instability of offshore cables during installation, in: ASME 36th International Conference on Ocean, Offshore and Arctic Engineering (OMAE2017), 25-30 June 2017, Trondheim, Norway. 
[22] Simo JC, Vu-Quoc L. On the dynamics in space of rods undergoing large motions - A geometrically exact approach. Comput Methods Appl Mech Eng 1988;66:125-161.

[23] Sobottka G, Lay T, Weber A. Stable integration of the dynamic Cosserat equations with application to hair modeling. J WSCG 2008;16:73-80.

[24] Thompson JMT, Champneys AR. From helix to localized writhing in the torsional postbuckling of elastic rods. Proceedings of the Royal Society of London A 1996;452:117-138.

[25] Tucker RW, Wang C. Torsional vibration control and Cosserat dynamics of a drill-rig assembly. Meccanica 2003;38:143-159.

[26] van der Heijden GHM, Neukirch S, Goss VGA, Thompson JMT. Instability and self-contact phenomena in the writhing of clamped rods. Int J Mech Sci 2003;45:161-196.

[27] Xie X, Zhang C, Liu H, Liu C, Jiang D, Zhou B. Continuous-mass-model-based mechanical and electrical co-simulation of SSR and its application to a practical shaft failure event. IEEE Transactions on Power Systems 2016;31:5172-5180. 